\title{
Functional imaging in muscular diseases
}

\author{
Erick Amarteifio • Armin Michael Nagel • \\ Hans-Ulrich Kauczor • Marc-André Weber
}

Received: 9 January 2011 /Revised: 10 April 2011 / Accepted: 9 June 2011 / Published online: 28 June 2011

(C) European Society of Radiology 2011

\begin{abstract}
Objective The development of morphological and functional imaging techniques has improved the diagnosis of muscular disorders.

Methods With the use of whole-body magnetic resonance imaging (MRI) the possibility of imaging the entire body has been introduced. In patients with suspected myositis, oedematous and inflammatory changed muscles can be sufficiently depicted and therefore biopsies become more precise.

Results Functional MR methods visualise different aspects of muscular (patho)physiology: muscular sodium $\left(\mathrm{Na}^{+}\right)$ homeostasis can be monitored with ${ }^{23} \mathrm{Na} \mathrm{MRI}$; the muscular energy and lipid metabolism can be monitored using ${ }^{31} \mathrm{P}$ and ${ }^{1} \mathrm{H}$ MR spectroscopy. ${ }^{23} \mathrm{Na}$ MRI has reached an acceptable value in the diagnosis and follow-up of patients with muscular $\mathrm{Na}^{+}$channelopathies that are characterised by myocellular $\mathrm{Na}^{+}$overload and consecutive muscle weakness. Besides MRI, low mechanical index contrast-enhanced ultrasound (CEUS) methods have also been introduced. For evaluation of myositis, CEUS is more efficient in the diagnostic work-up than usual b-mode
\end{abstract}

E. Amarteifio $(\bowtie) \cdot H .-U$. Kauczor $\cdot$ M.-A. Weber

Department of Diagnostic and Interventional Radiology,

University Hospital of Heidelberg,

INF 110 ,

69120 Heidelberg, Germany

e-mail: erick.amarteifio@med.uni-heidelberg.de

E. Amarteifio $\cdot$ M.-A. Weber

Department of Radiology, German Cancer Research Center,

Heidelberg, Germany

\section{A. M. Nagel}

Department of Medical Physics in Radiology,

German Cancer Research Center,

Heidelberg, Germany ultrasound, because CEUS can detect the inflammatoryinduced muscular hyperperfusion in acute myositis. Moreover, the arterial perfusion reserve in peripheral arterial disease can be adequately examined using CEUS.

Conclusion Modern muscular imaging techniques offer deeper insights in muscular (patho)physiology than just illustrating unspecific myopathic manifestations like oedematous or lipomatous changes, hypertrophy or atrophy.

Keywords Imaging · MRI · Magnetic resonance ·

Ultrasound $\cdot$ Muscular diseases

\section{Introduction}

Various causes, like neurogenic, metabolic, dystrophic or inflammatory mechanisms, as well as muscular channelopathies leading to disorders of the muscle cell membrane, may potentially cause muscular diseases. Although muscular diseases are a heterogeneous group, in most cases of progressive disease, the result is a focal or general muscular weakness. Unfortunately, muscular weakness is a very unspecific symptom that often does not allow conclusions to be drawn about the origin of the paresis. Standard neuromuscular literature suggests that radiological imaging would play only an inferior role because of absent pathognomonic findings [1]. Why does radiological imaging hold such a low significance in the case of muscular diseases? Routine protocols of magnetic resonance imaging (MRI) only show morphology and demonstrate oedematous or lipomatous changes, atrophy or hypertrophy (Fig. 1). However, these morphological changes are not very disease-specific [2-6]. Apparent changes like consecutive muscular haematoma after blunt trauma can be nicely verified, but the huge and heterogeneous group of 


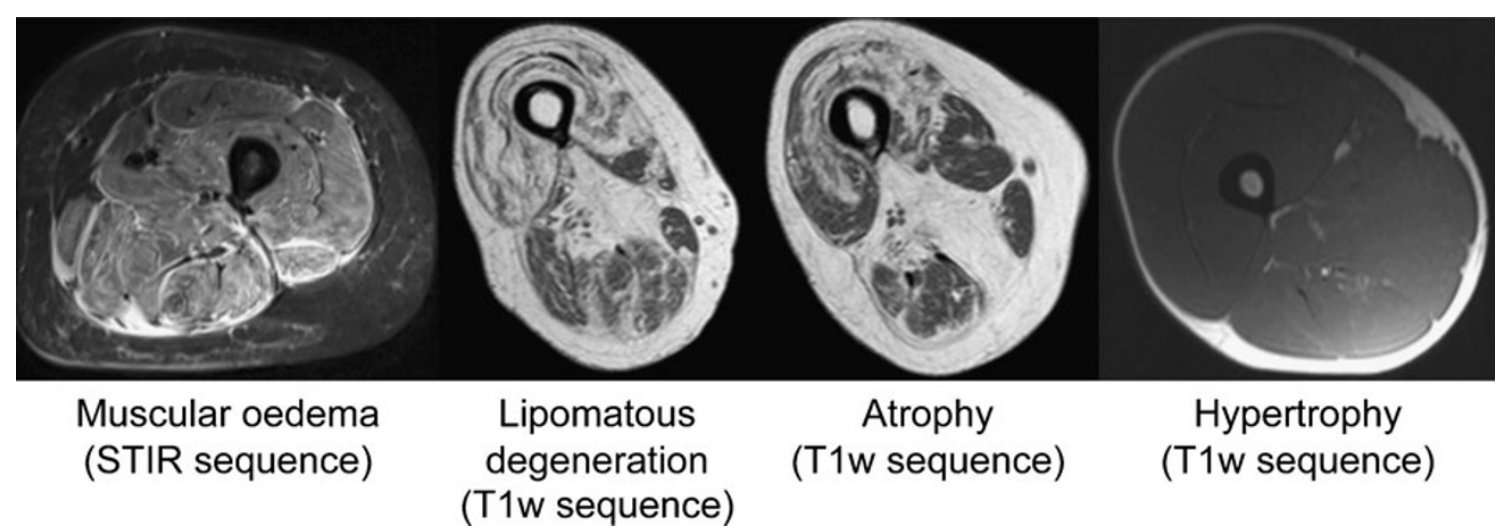

Fig. 1 Typical pathological changes in muscular tissue shown in standard proton $\left({ }^{1} \mathrm{H}\right)$ MRI

myopathies based upon different origins cannot be further differentiated because the underlying (patho)physiology is not demonstrated. The following short review introduces examples of modern functional imaging methods for the evaluation of muscular diseases with emphasis on pathophysiological fundamentals.

\section{Whole-body MRI}

Whole-body MRI allows the entire human body to be investigated within one single examination. It is necessary for the patient to be completely covered with coils (Fig. 2a). The examination lasts up to approximately $60 \mathrm{~min}$, but the duration depends on the protocol and sequence design used; for example, turbo-spin-echo and gradient echo sequences require comparatively less time than spin-echo sequences. For the diagnosis of muscular disorders, axial and coronal T1weighted sequences as well as axial and coronal short-tau inversion recovery (STIR) sequences are useful (Fig. 2b, c). Contrast media are not needed in most cases. One of the main advantages of whole-body MRI in the field of muscular disorders is biopsy targeting: when a biopsy is planned, adequate targets can regularly be found using MRI as oedematous muscular changes, e.g. in suspected dermatositis or polymyositis [7]. The diseases have to be diagnosed quickly, because they can be treated successfully with immunosuppressive therapy, contrary to many other muscular diseases. Unfortunately, they lead to irreversible muscular weakness when the diagnosis is missed. A central problem is that the muscles may be affected focally, and false-negative biopsy findings are found in up to $25 \%$ in random biopsies instead of image guidance. Common findings in MRI of an active inflammatory process are oedematous changes of the muscular tissue that can be-more reliably than with clinical examination or electromyography-identified and mapped using whole-body MRI $[8,9]$. With a combination of fatsuppressed T2-weighted sequences and T1-weighted sequences, fatty degenerated muscular tissue can be nicely differentiated from oedematous affected muscular tissue as a hint for acute inflammation. These muscle regions showing oedematous changes, which are potentially caused by inflammation, should be targeted. Therefore, biopsy results will be improved when performing biopsy with imaging guidance.

However, the pathological changes of muscular tissue are less specific. For example, rhabdomyolysis, muscular dystrophy or acute neuromuscular denervation may appear with muscular oedematous changes, too. Generally, oedematous changes give a hint for an acute process, whereas lipomatous degeneration is observed in chronic stages of disease.

Another advantage of whole-body MRI is its potential to show patterns of muscle involvement that are in favour of a specific myopathy. Also, better than with clinical examination, e.g. by using the Medical Research Council (MRC) score for paresis, subtle involvement of muscle groups can be detected [10].

For several forms of cancer, whole-body MRI is regularly used for the detection or control of metastases. For example, in patients with multiple myeloma, metastases involving connective tissue or muscle can regularly be identified (Fig. 3)

\section{${ }^{23} \mathrm{Na} \mathrm{MRI}$}

Conventional MRI uses the gyration of protons $\left({ }^{1} \mathrm{H}\right)$, basically of water and carbohydrate compounds, for image acquisition. Innovative techniques like ${ }^{23} \mathrm{Na}$ MRI offer the possibility of detecting sodium ions $\left(\mathrm{Na}^{+}\right)$. In a healthy muscle cell, the $\mathrm{Na} / \mathrm{K}$-ATPase moves $\mathrm{Na}^{+}$out of the cytoplasm and $\mathrm{K}^{+}$into the cytoplasm and thus contributes to maintaining a constant membrane potential and an $\mathrm{Na}^{+}$ concentration gradient. In healthy tissue, the extracellular sodium concentration $\left([\mathrm{Na}+]_{\mathrm{ex}}=145 \mathrm{mM}\right)$ is about tenfold higher than the intracellular concentration $\left([\mathrm{Na}+]_{\mathrm{in}}=10\right.$ $15 \mathrm{mM})$ [11]. ${ }^{23} \mathrm{Na}$ MRI allows volume- and relaxationweighted measurements of these $\mathrm{Na}^{+}$compartments, non- 
a

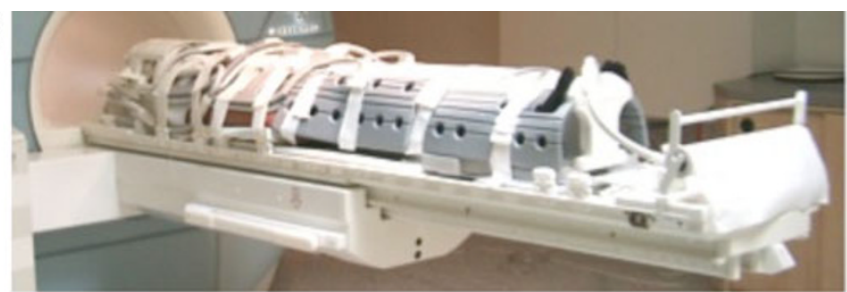

b

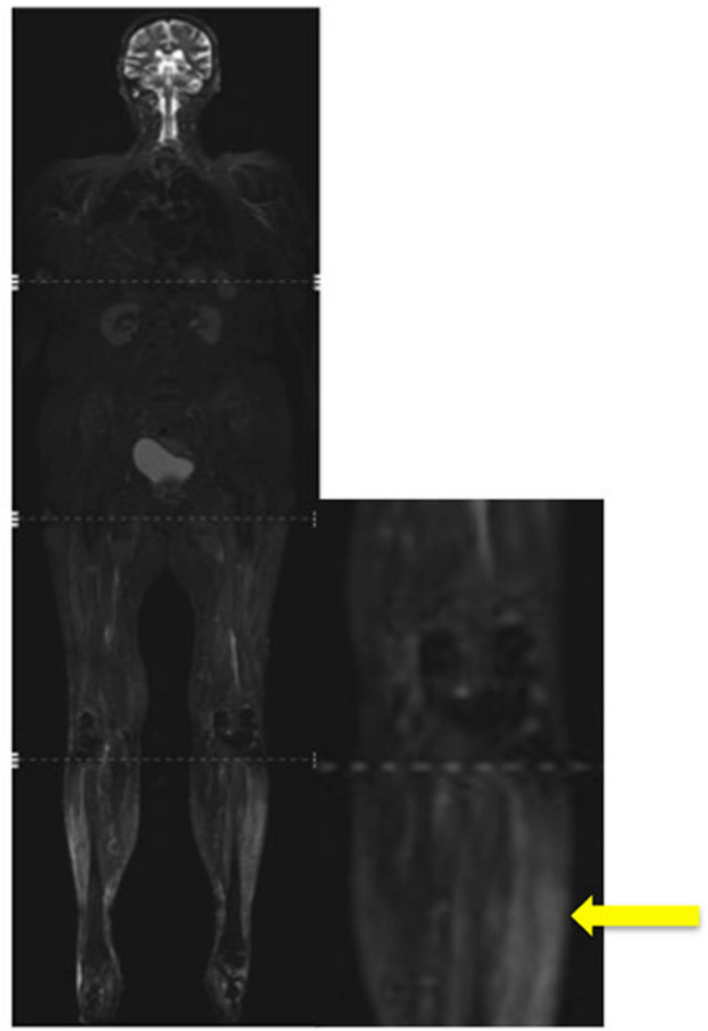

Fig. 2 a Example of a completely covered patient during a wholebody MRI examination. b Whole-body MRI of a 67-year-old patient with sarcoidosis-associated chronic myositis. The arrow clearly shows oedematous changes within the enlarged image of the left fibularis

invasively. The membrane potential is necessary to allow for a contraction of muscle cells. Muscular $\mathrm{Na}^{+}$channels within the cell membrane provide auxiliary control of $\mathrm{Na}^{+}$ homeostasis. In several groups of muscle diseases, the muscular sodium channelopathies, patients are affected from an incomplete inactivation of these muscular $\mathrm{Na}^{+}$ channels. The resulting $\mathrm{Na}^{+}$leak leads to an inward $\mathrm{Na}^{+}$ current that causes an ongoing depolarisation of muscle fibres and an increase in the intracellular $\mathrm{Na}^{+}$concentration. This leads to an elevated total $\mathrm{Na}^{+}$concentration compared with healthy muscle tissue. ${ }^{23} \mathrm{Na}$ MRI is able to noninvasively measure this pathological increase in the $\mathrm{Na}^{+}$ concentration. However, ${ }^{23} \mathrm{Na}$ MRI is associated with several challenges. First, the physical NMR sensitivity of ${ }^{23} \mathrm{Na}$ is only about $9 \%$ of the sensitivity of ${ }^{1} \mathrm{H}$. Considering a 1,000 - to 5,000 -fold lower in vivo concentration

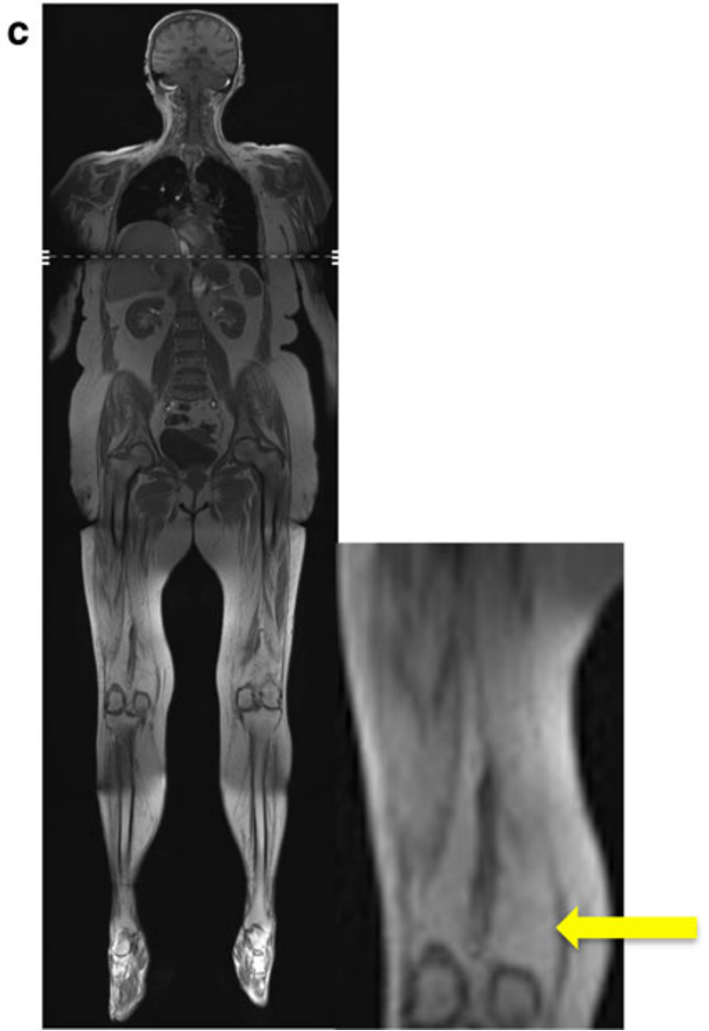

muscle using fat-saturated sequences. $\mathbf{c}$ Whole-body MRI of the same patient as in b: standard T1-weighted sequences clearly show lipomatous changes. The arrow within the enlarged image indicates lipomatous degeneration of the right semimembranosus muscle

compared with ${ }^{1} \mathrm{H}$, this leads to an 11,000 - to 55,000 -fold lower ${ }^{23} \mathrm{Na}$ signal. Second, the ${ }^{23} \mathrm{Na}$ signal in vivo decays bi-exponentially, with a fast $(0.5-3.0 \mathrm{~ms})$ and a slow (15$30 \mathrm{~ms}$ ) component. To measure the total ${ }^{23} \mathrm{Na}$ signal, sequences with ultra-short echo times are necessary [12]. Furthermore, acquisition techniques that combine both short echo times and high signal-to-noise ratio efficiency, such as twisted projection imaging [13] or density-adapted sampling [14] are favourable for ${ }^{23} \mathrm{Na}$ MRI. Moreover, specific hardware and software are needed; for example, double resonant coils that are able to work with the resonance frequency both of sodium nuclei $(16.8 \mathrm{MHz}$ at 1.5 Tesla; $78.6 \mathrm{MHz}$ at 7 Tesla) and protons $(63.6 \mathrm{MHz}$ at 1.5 Tesla; $300 \mathrm{MHz}$ at 7 Tesla). It is a further challenge to discriminate between the intracellular and extracellular amount of sodium via non-invasive techniques like MRI. 
Fig. 3 Whole-body MRI of a 45-year-old patient with multiple myeloma. The STIR sequence clearly shows a bone metastasis located in the left os ilium and the perifocal muscular reaction depicted as oedematous changes

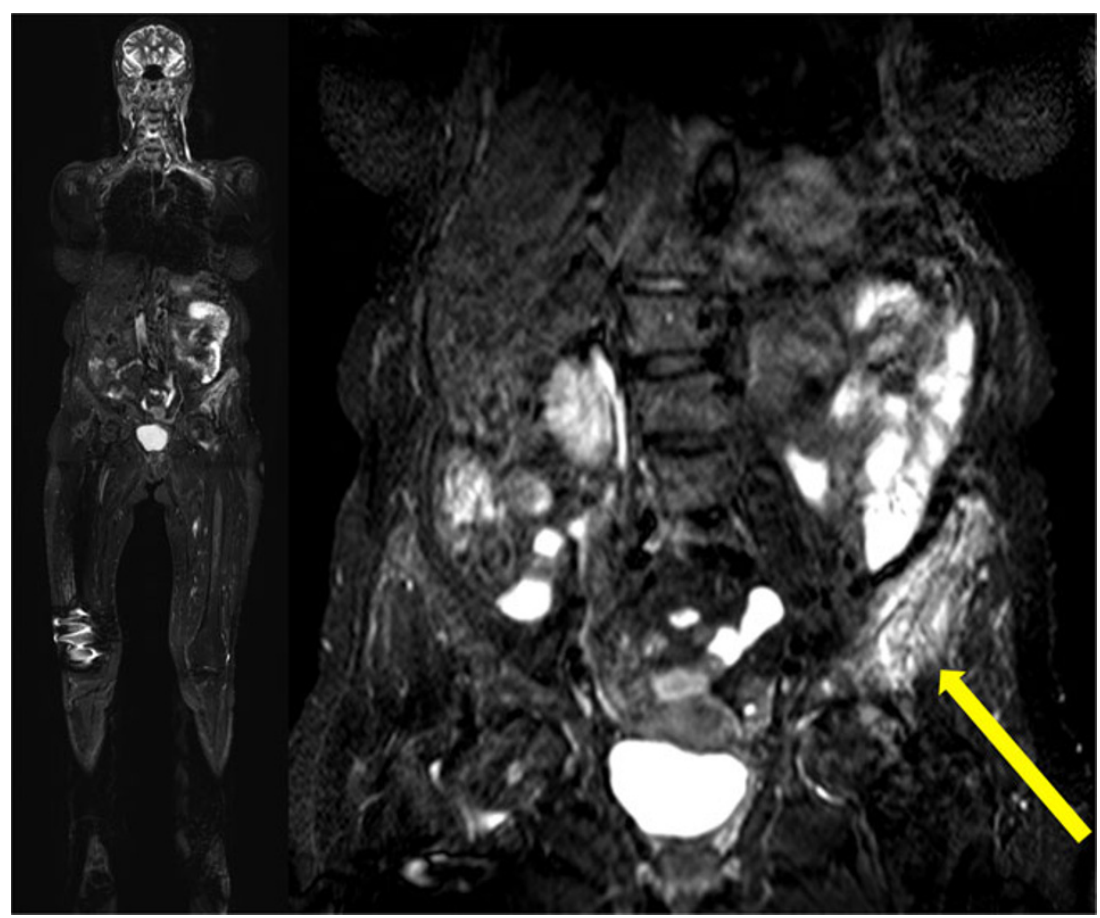

Paramagnetic shift reagents allow for a clear separation between intracellular and extracellular sodium [15]. Unfortunately, they cannot be applied in humans because of their toxicity. Current research in ${ }^{23} \mathrm{Na}$ MRI demonstrated the possibility of reducing the signal from extracellular sodium compartments, such as in vasogenic oedema. A ${ }^{23} \mathrm{Na}$ inversion recovery sequence was developed to reduce the ${ }^{23} \mathrm{Na}$ signal received from vasogenic oedema to achieve a weighting of the intracellular ${ }^{23} \mathrm{Na}$ amount [16]. ${ }^{23} \mathrm{Na} \mathrm{MRI}$ has effectively achieved value in the radiological management of muscular sodium channel diseases $[17,18]$. In the affected patients, an autosomal-dominant bequeathed defect of muscular $\mathrm{Na}^{+}$channels leads to a pathological $\mathrm{Na}^{+}$influx that causes intermittent or permanent muscular paresis as well as muscular stiffness (Fig. 4a) [19]. Typical triggers are exposure to cold or ingestion of potassium-rich food. The diseases appear rarely, examples would be hyperkalaemic and hypokalaemic periodic paralysis or paramyotonia congenita. ${ }^{23} \mathrm{Na}$ MRI is able to depict an intracellular muscular sodium accumulation simultaneous to development of muscular paresis or muscular stiffness (Fig. 4b). The sodium accumulation correlates well with the grade of paresis and is reproducible [18, 20]. Additionally, ${ }^{23} \mathrm{Na}$ MRI allows for therapy monitoring; for example, mexiletine reduces myoplasmic sodium overload in patients with paramyotonia congenita. With ${ }^{23} \mathrm{Na} \mathrm{MRI}$, the decrease in myoplasmic $\mathrm{Na}^{+}$content becomes visible non-invasively. Another example is hypokalaemic periodic paralysis that goes along with intramuscular $\mathrm{Na}^{+}$accumulation. The sodium overload causes muscle oedema and weakness in these patients, and it now can be treated specifically with acetazolamide [17]. The muscular oedema as well as the muscular weakness will decrease under treatment, and the muscular strength will improve. Recently, the development of ${ }^{23} \mathrm{Na} \mathrm{MRI}$ at field strengths of 3-7 Tesla (standard are 1.5Tesla devices) allows for a more precise quantification of intracellular ${ }^{23} \mathrm{Na}$ homeostasis. In addition, ${ }^{23} \mathrm{Na}$ homeostasis may be examined more precisely in healthy volunteers as well as in patients, because initial findings indicate the high potential of ${ }^{23} \mathrm{Na}$ MRI at 7 Tesla (Fig. 5) [21].

\section{${ }^{31} \mathrm{P}$-magnetic resonance spectroscopy}

${ }^{31} \mathrm{P}$-magnetic resonance (MR) spectroscopy was introduced in the 1980s with the aim of evaluating the energy metabolism of muscular tissue. The phosphorous MR spectrum demonstrates several peaks of specific energy metabolites, like phosphocreatine, that mirrors a typical short-time energy deliverer, as well as anorganic phosphate that is a degradation product [22]. Moreover, adenosine-5'triphosphate, which is necessary for muscular contraction, as well as phosphodiester, can be extrapolated from the spectrum and quantified (Fig. 6a). When the chemical shift of anorganic phosphate to phosphocreatine is analysed, the intracellular $\mathrm{pH}$ value can be calculated. These techniques can be used successfully in vivo during muscular exercise and can depict a decrease in phosphocreatine during physical trials [23]. The technique offers a useful diagnostic tool; for example, in patients suffering from the glycogenosis McArdle's disease, the muscular glycolysis is disturbed, and 


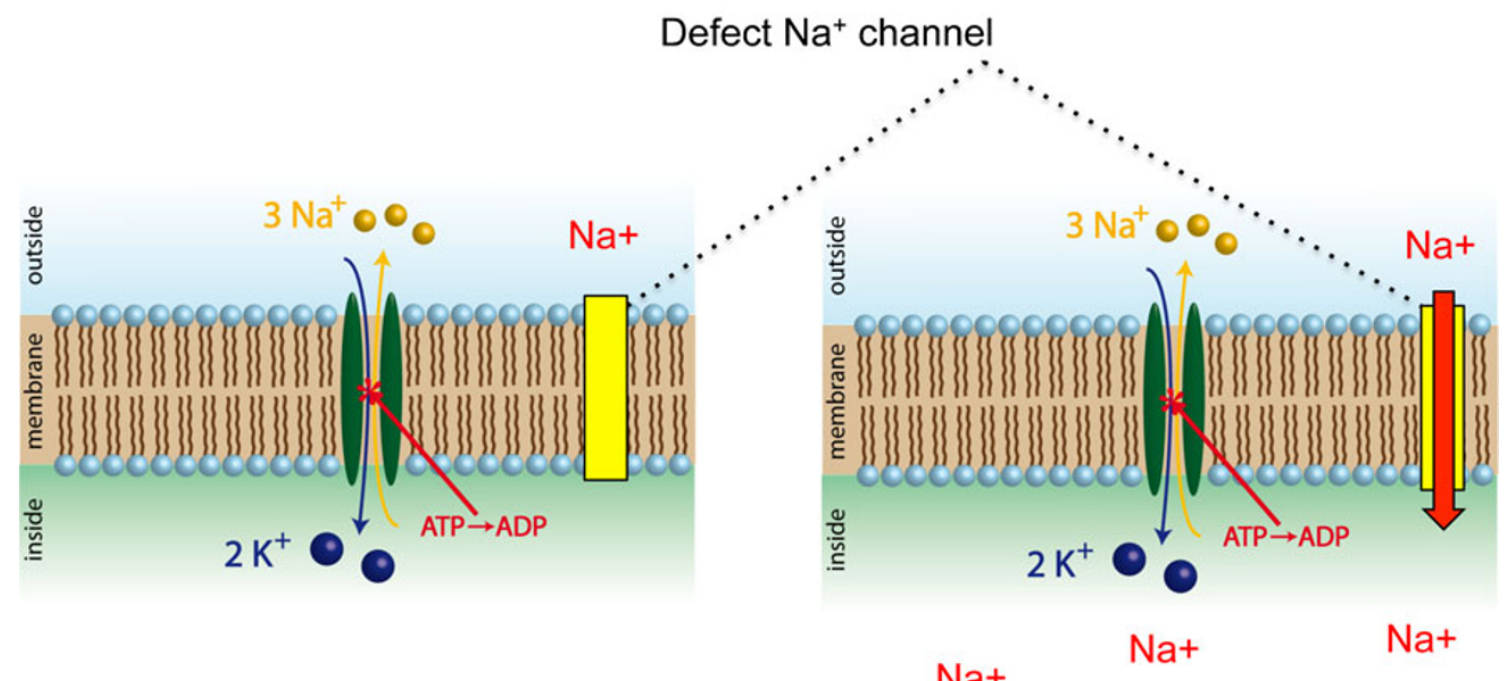

\section{Provocation by cooling leads to intracellular $\mathrm{Na}^{+}$accumulation}

$\mathrm{Na}+$

\section{$\mathrm{Na}+$}

$\mathrm{Na}+\mathrm{Na}+$

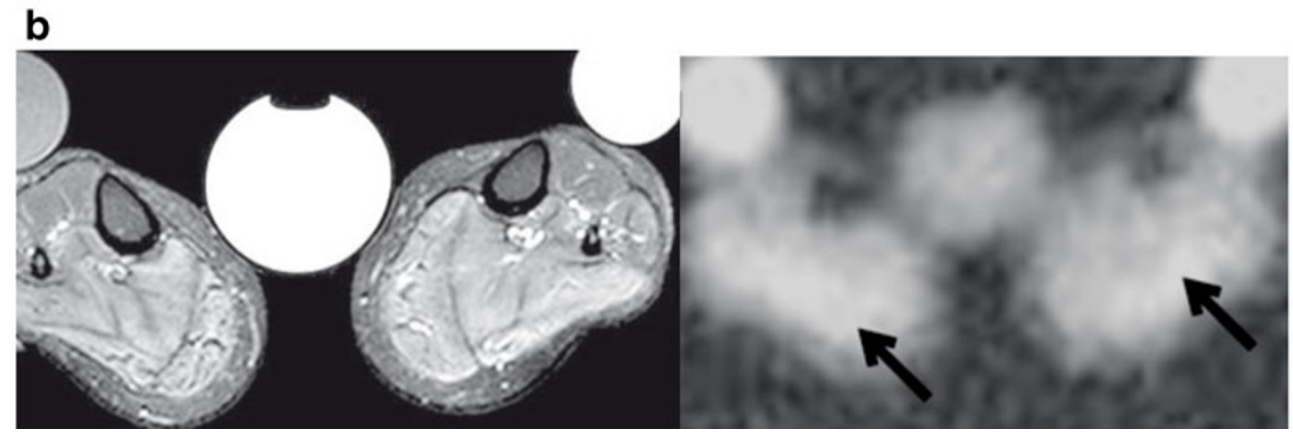

Fig. 4 a In muscular sodium channel diseases, a defective sodium channel within the muscular cell membrane leads to a pathological influx of $\mathrm{Na}^{+}$after provocation with typical triggers, e.g. exposition to cold. Results are muscular weakness or muscular stiffness. b STIR weighted ${ }^{1} \mathrm{H}$ imaging shows elevated signal in the lower leg muscles of this patient suffering from hypokalaemic periodic paralysis type 1 . The intracellular $\mathrm{Na}^{+}$accumulation can be visualised nicely with ${ }^{23} \mathrm{Na}$ (arrows). Observe the reference tubes: $0.3 \% \mathrm{NaCl}$ solution in the middle, $0.3 \% \mathrm{NaCl}$ bound with agarose gel on the left-hand side, $0.6 \%$ $\mathrm{NaCl}$ solution on the right-hand side the physiological decrease of $\mathrm{pH}$ value due to lactate production does not take place. This can reliably be demonstrated in patients with glycogenosis/McArdle's disease using ${ }^{31} \mathrm{P}-\mathrm{MR}$ spectroscopy [24, 25]. Several research groups tried to find a specific pattern using ${ }^{31} \mathrm{P}-\mathrm{MR}$ spectroscopy, but the muscular degeneration finally leads to lipomatous conversion and consecutive loss of high-energy phosphates within the spectrum that can finally be observed in all chronic myopathies. Moreover, the cross-sectional area of muscular fibres correlates with the amount of phosphocreatine and adenosine-5'-triphosphate. This correlation makes sense, as both metabolites are necessary for muscular contraction. Further developed ${ }^{31} \mathrm{P}-\mathrm{MR}$ spectroscopy-related imaging techniques allow for a spatial illustration of the phosphocreatine signal received and thus a two-dimensional illustration of the muscular energy level [26].

\section{Proton $\left({ }^{1} \mathrm{H}\right)$ MR spectroscopy}

This method delivers the opportunity to quantify intramyocellular lipids [27]. Further interesting metabolites are phosphocreatine, choline as a marker for the rebuilding of the cell membrane, or extramyocellular lipids that are present within the lipomatous intermuscular connection tissue and the subcutis and are regarded as a contaminator of the intramyocellular lipids in a spectrum (Fig. 6b). But intramyocellular lipids show a highly inter-individual variability and it is obvious that different physical conditions of muscles obtain different concentrations of this lipid fraction. Therefore, it sounds a paradox that athletes show a high level of intramyocellular lipids and a high insulin sensitiveness of their muscular tissue. However, the intramyocellular lipids serve as energy donators for the 
Fig. $5{ }^{23} \mathrm{Na}$ MRI at $3 \mathrm{~T}$ $(\mathrm{TE}=0.2 \mathrm{~ms})$ and $7 \mathrm{~T}(\mathrm{TE}=$ $0.55 \mathrm{~ms})$. Transverse and coronal slices of the lower legs in a healthy volunteer. Observe the significantly improved signal-to-noise ratio at $7 \mathrm{~T}$
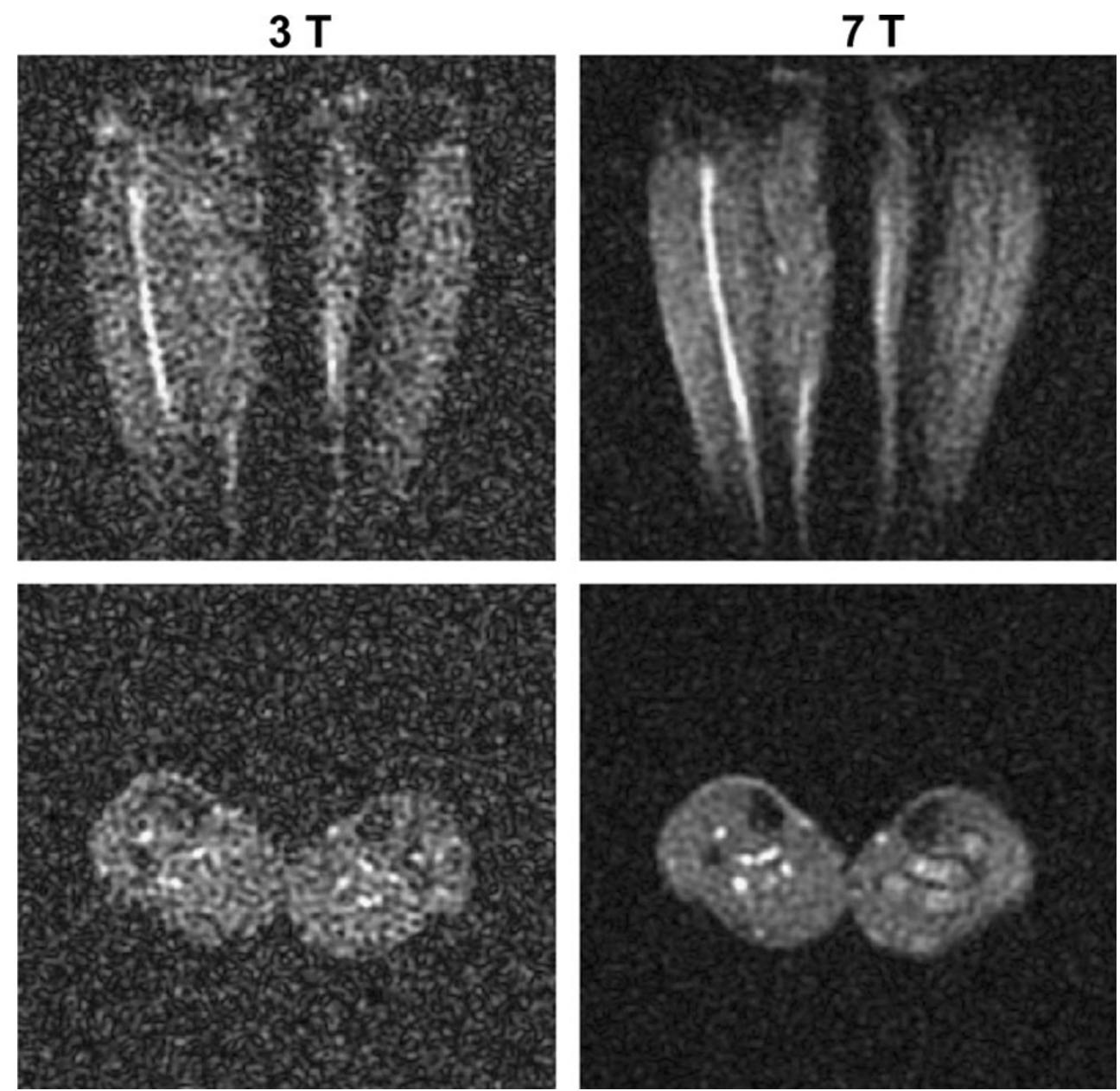

muscular mitochondria. Physical work leads to a decrease in intramyocellular lipids. Interestingly, untrained patients suffering from type-2 diabetes with peripheral muscular insulin resistance show a high level of intramyocellular lipids too $[28,29]$. In the end, many parameters influence the level of intramyocellular lipids, e.g. dietary nutrition, sex, gender, body mass index and the oxygen content of tissue. Additionally, modern spectroscopic techniques deliver the possibility of spatially visualising the evaluated resonance peaks with the aid of parametric colour maps. Several groups are researching ${ }^{13} \mathrm{C}$-MR spectroscopy. The nucleus plays an important part in muscle physiology and pathophysiology $[30,31]$. A central problem is that the MRI need special equipment like broadband transmitters and receivers to allow for ${ }^{13} \mathrm{C}$-MR spectroscopy. Nevertheless, a further development of this technique may be expected in the future.

\section{Blood oxygen level-dependent MRI}

Blood oxygen level-dependent (BOLD) MRI mirrors the local ratio of oxyhaemoglobin and deoxyhaemoglobin. T2*-weighted functional MRI sequences are in common use for BOLD MRI. The signal acquisition is based upon susceptibility effects and subsequent MRI signal changes in muscular tissue, caused by the paramagnetic effect of deoxyhaemoglobin. Oxyhaemoglobin, in turn, is diamagnetic. For muscular tissue, in particular, the signal is influenced by the microvascular density, blood flow and volume, and oxygen extraction rate [32-36]. Experiments evaluating the BOLD response of muscular tissue during post-occlusive reactive hyperaemia showed a steep increase in the T2* signal, followed by a non-linear signal decrease. In patients with peripheral arterial disease (PAD), the T2* signal increase during post-occlusive reactive hyperaemia is a lesser step and delayed. Moreover, a significant age-dependent difference in the rate, intensity and recovery of the post-occlusive muscle BOLD signal was revealed [37, 38].

\section{Arterial spin labelling MRI}

Arterial spin labelling (ASL) is based upon magnetic labelling of moving blood particles of a certain slice by an inversion pulse at the first step, and read-out of the labelled blood particles during arrival in the slice of interest (for example, the capillary bed) at the second step [39]. Before acquisition of the image with labelled blood 
a

Phosphor ( $\left.{ }^{31} \mathrm{P}\right)$ MR-spectrum

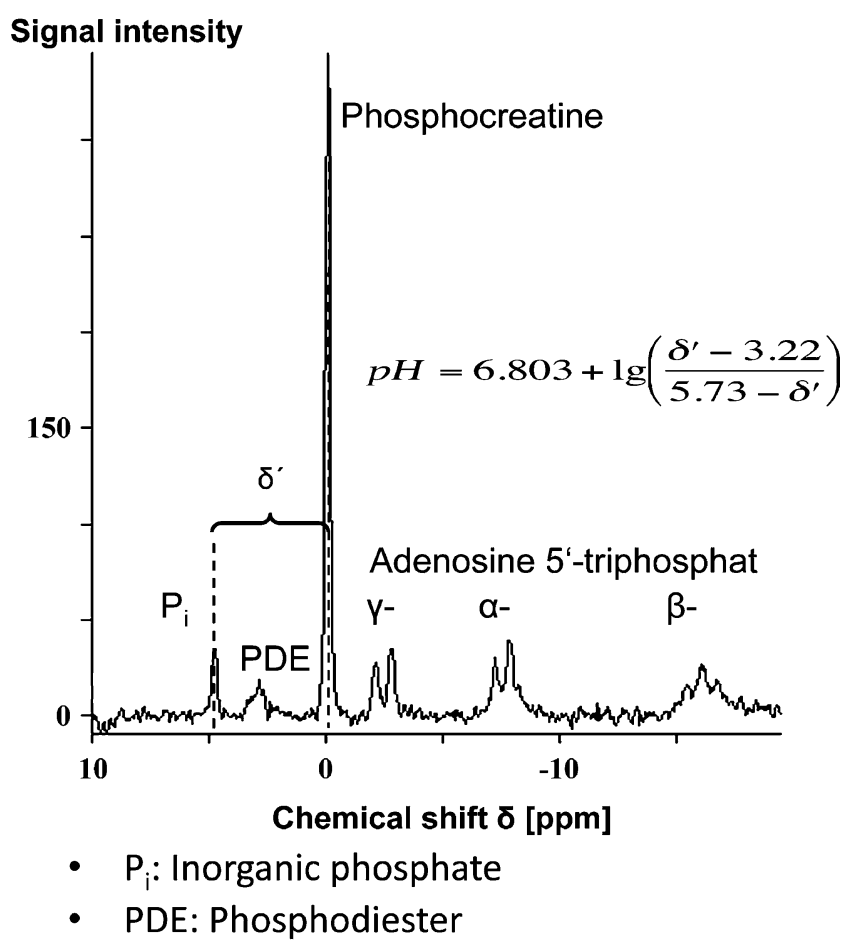

b Proton $\left({ }^{1} \mathrm{H}\right)$ MR-spectrum

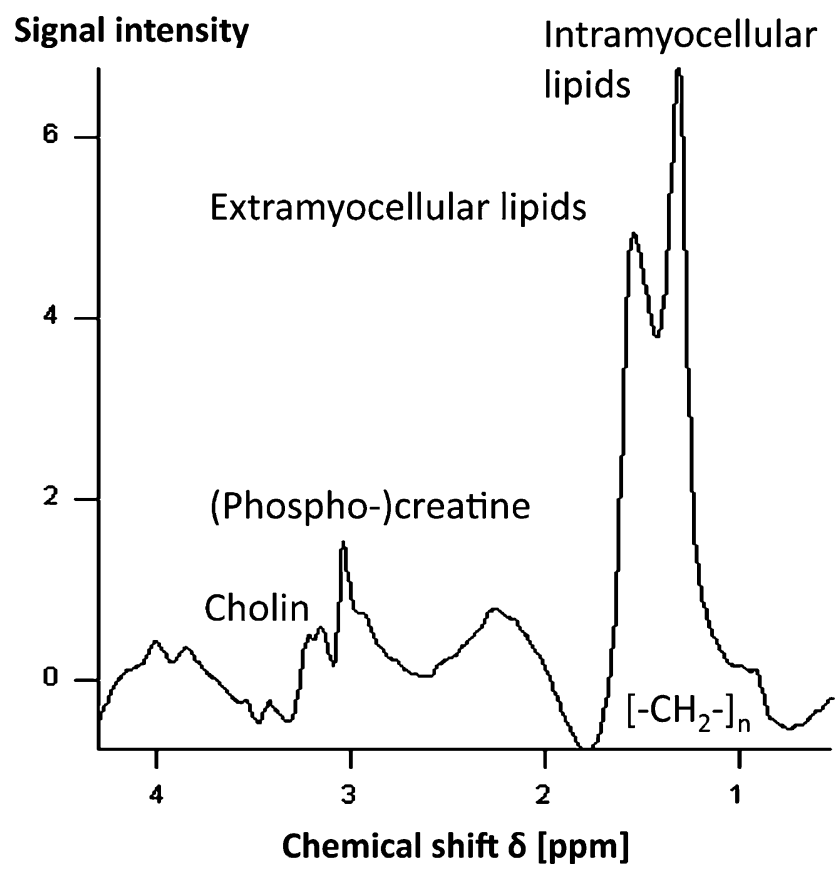

Fig. 6 Typical metabolites in the muscular ${ }^{31}$ phosphorous MR spectrum (a) and muscular ${ }^{1} \mathrm{H}-\mathrm{MR}$ spectrum (b)

particles, an image of the same region without labelled blood particles is acquired and serves as a control. The difference between the labelled and the control image yield the perfusion-weighted image (Fig. 7). The received signal increases linearly proportional to the underlying tissue perfusion [40, 41]. An advantage is the illustration of local perfusion without the need for contrast media. A disadvantage is the comparatively long examination time. The technique has reached a level of clinical acceptance for quantification of muscular perfusion with MRI [39, 41]. Moreover, the development of arterial spin labelling techniques at ultra-high field MRI showed that the signalto-noise ratio and thus image quality is enhanced with an increase in field strength [42] — which will additionally improve the frequency of clinical implementation.

MR elastography

With MR elastography (MRE) a quantitative measure of mechanical elasticity of tissue and muscles is possible. The technique allows for visualisation of disturbed fibre characteristics due to pathological changes within the tissue. For example, cancer, inflammation and fibrosis may change the local tissue elasticity. Image acquisition obtains several steps. First, shear waves within the examined tissue are generated. Second, the propagation of the induced waves is depicted with MRI. Third, a postprocessing of the depicted shear wave images is performed and quantitative maps of tissue elasticity are produced. For skeletal muscle, several approaches in MRE exist. For example, MRE showed that neuromuscular diseases (i.e. childhood poliomyelitis, spastic and flaccid paraplegia) have an influence on muscle elasticity [43]. Moreover, MRE can be used for quantification of muscle stiffness caused by immobilisation [44] and thus has the potential to visualise effects of rehabilitation in patients with loss of mobility. In conclusion, MRE delivers important information about the local or general fibre physiology and pathology; it can be predicted that MRE will reach a higher level of clinical acceptance in the future.

Diffusion-weighted imaging and diffusion tensor imaging

With diffusion-weighted imaging (DWI) the mobility of water molecules (and therefore the mobility of ${ }^{1} \mathrm{H}$ protons) can be visualised. The fundamental hypothesis is that the mobility of water molecules within diseased tissue (for example, cancer-involved or inflammatory tissue) differs from that of normal tissue. For image acquisition, the spins of ${ }^{1} \mathrm{H}$ protons are activated and the resulting echo is recorded. In diseased tissue with decreased ability of diffusion, the activated ${ }^{1} \mathrm{H}$ protons obtain decreased mobility and thus remain at almost the same place. The result is a high intensity of regions with disturbed diffusion on DWI images. The potential of DWI for brain and stroke 

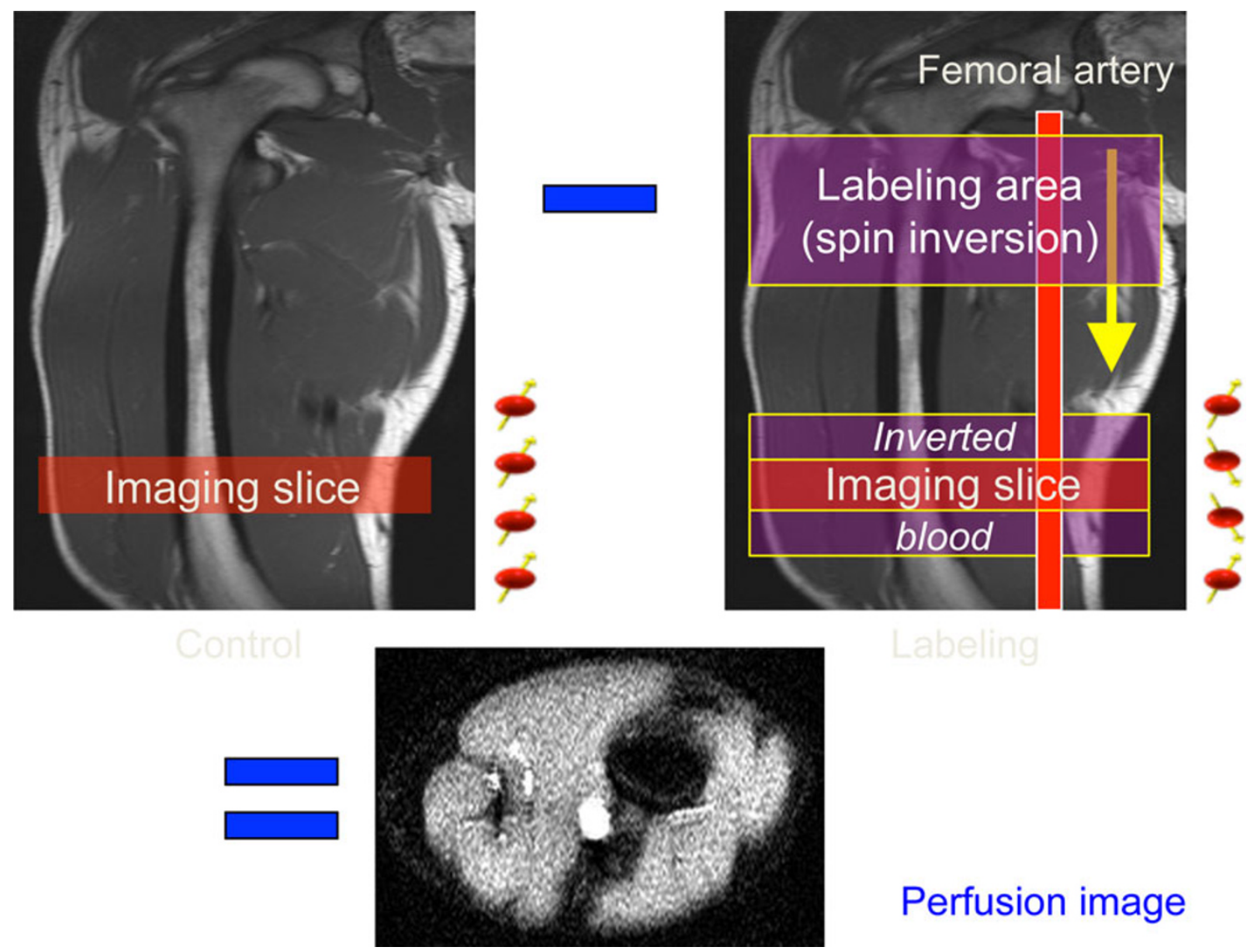

\section{Perfusion image}

Fig. 7 Description of arterial spin labelling. Previously inverted magnetisation enters the slice of interest and is exchanged with normal tissue magnetisation. The resulting image intensity is a measure of local tissue perfusion

imaging has already been well demonstrated [45-47]. Diffusion tensor imaging (DTI) is a further development of DWI, where the direction of diffusion is captured. As a benefit, tractographies, for example, of neuronal structures like nerve fibres, can be reconstructed. Development of DWI and DTI for examination of the musculoskeletal system is still just beginning, but the first results are promising [48, 49]. Physiological and impaired muscle function can be sufficiently examined by using diffusion tensor imaging. As DTI values are related to the state of muscle contraction, the technique allows for a non-invasive measure of muscle elasticity and its capability of contraction [50]. A precise quantification of muscular DTI seems to be reproducible even in different individuals, as age or gender has no effect on water diffusivity values [51]. With an emphasis on in vivo MR tractography, the anatomical structure and micro-architecture of muscle fibres can be visualised [52]. DTI offers the opportunity to qualitatively determine the impacting force vectors. For example, in patients with lateral patellar dislocation, the visualisation of force vectors delivers important information about the static and dynamic constitution of the knee joint [53]. Not only muscles, but also nerves are visualised by functional MRI.
Recent research in neuro-imaging attested DTI to be an excellent tool for detecting injuries or entrapments of peripheral nerves and their recovery after trauma [54]. The deranged and recovered micro-architecture of the peripheral nerves itself becomes visible, whereas conventional sequences like STIR only provide secondary information, such as consecutive oedema.

\section{Real-time ultrasound elastography}

With ultrasound elastography, the relative tissue rigidness can be visualised. Therefore, the technique allows for a more objective quantification of the findings gained from conventional palpation by the physician. Analogous to the duplex ultrasound, the colour-coded map that mirrors the tissue rigidness is overlaid on the B-mode ultrasound image. The performance of ultrasound elastography for detection of suspicious breast lesions, pancreatic lesions or suspicious lymphatic nodules had been approved before [55-57]. In the case of muscular diseases, ultrasound elastography delivers additional information about the local condition of the muscle, because the local muscle rigidness may be disturbed in several muscular diseases. Moreover, 
the technique can be used to localise parts of diseased muscle and thus alleviate muscle biopsies.

\section{Contrast-enhanced ultrasound (CEUS)}

Usually, B-mode ultrasound is the first practiced and the most frequently used examination to evaluate muscular disorders. The outcome of sports accidents like ruptures of muscular fibres, haematomas, ruptures of ligaments or extrusion can be well demonstrated [58]. The development of ultrasound over the last few years has made it possible to visualise (patho)physiological information about muscular microcirculation. Ultrasound contrast media are stabilised microbubbles that stay within the vasculature and thus can be used to evaluate the capillary muscular perfusion.

Ultrasound contrast media of the first generation consist of microbubbles that are burst with the use of high-energy ultrasound pulses. During the burst, an ultrasound signal is emitted, a so-called stimulated acoustic emission (SAE), and can be received. A disadvantage of first-generation ultrasound contrast media was that an evaluation of local perfusion in real time was not possible. For example, examination during muscular exercise could not be performed.

Second-generation ultrasound contrast media like SonoVue (Bracco Imaging, Milan, Italy) are not destroyed by ultrasound waves but are put into oscillation. Using this technique, increased muscular tissue perfusion in correlation with an increased number of capillaries can be evaluated in real time. For example, endurance-trained athletes obtain an increased muscular tissue perfusion during rest in combination with a high density of capillaries compared with non-trained volunteers. Moreover, the quantified local blood volume within the selected ultrasound slice correlates with the aerobic capacity and with the perfusion evaluated with venous occlusion plethysmography [59]. Also, changes in the micro-
Fig. 8 Examples of typical signal-intensity-over-time curves of the dynamic CEUS examination. a A rapid increase in the measured CEUS signal was observed after release of arterial occlusion in a healthy volunteer, while the timeintensity curve of a patient with peripheral arterial disease (walking distance, $100 \mathrm{~m}$ ) increased more slowly with a delayed and less high maximum after provocation (b)
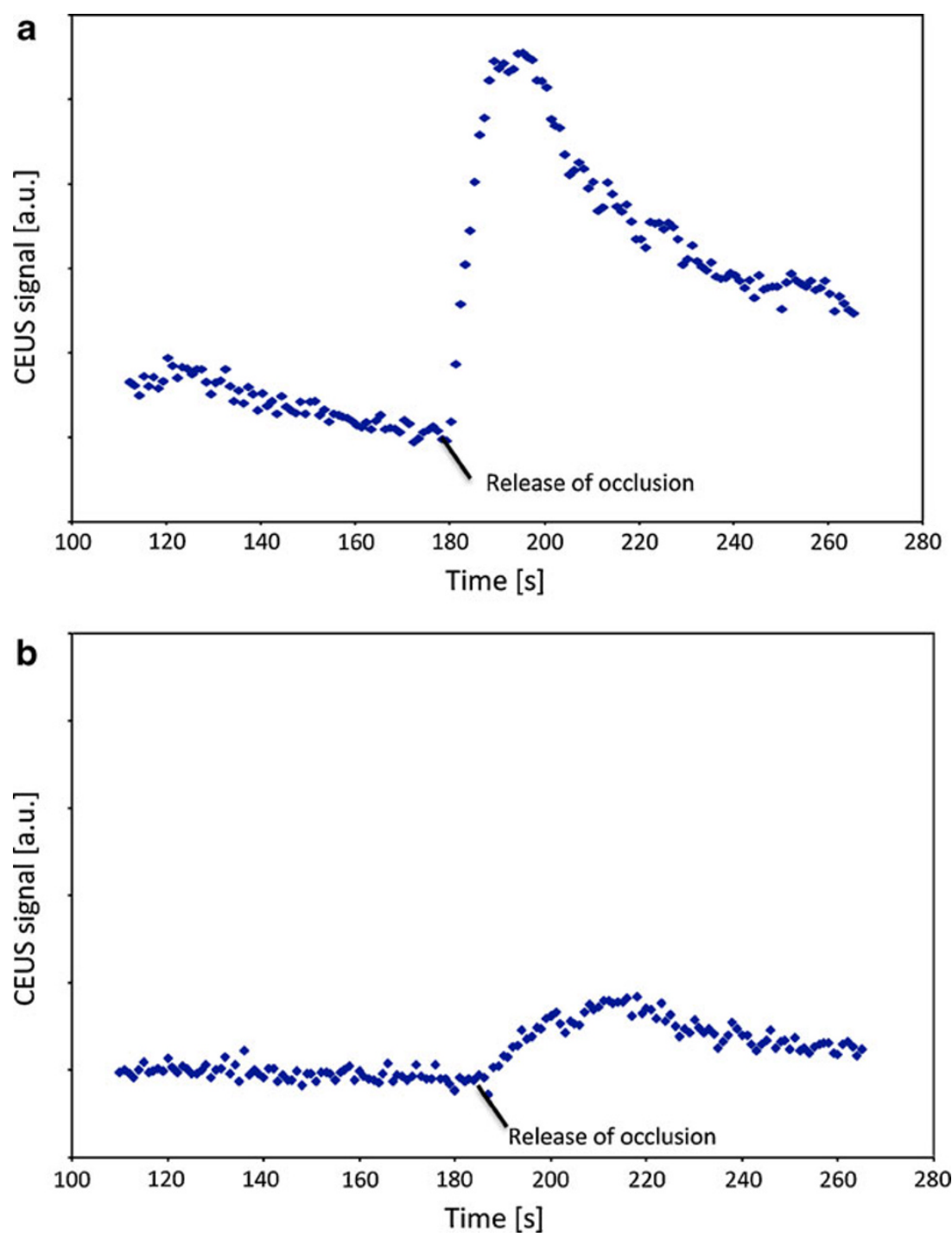
circulation of skeletal muscle during isometric exercise have become visible in real time [60]. This information allows for a better understanding of the physiological adaption of skeletal muscle due to physical strain or inflammation, and has the potential to improve exercise testing and prescription. In the case of confirmed dermatomyositis or polymyositis, a significantly higher muscular perfusion compared with healthy volunteers was shown [61]. Additionally, the oedematous muscular changes visible in B-mode ultrasound underline the suspicion of acute myositis. In the case of confirmed polymyositis, immunosuppressive therapy causes an increased muscular strength, a reduction in the creatinine kinase level and decreased muscular perfusion at rest.

Special ultrasound techniques allow for the detection of oscillating microbubbles, separation from muscular tissue and deliver the opportunity to assess muscular tissue perfusion in real time. Depending on the grade of isometric physical exercise, reduced perfusion due to compression of muscular venules can be observed. At the end of physical exercise, an increased ultrasound signal can be detected because of reactive hyperperfusion. These findings showed that the resulting hyperperfusion depended on the grade of muscular exercise [59]. Moreover, the arterial perfusion reserve can be adequately examined with CEUS in combination with transient arterial occlusion. Initial findings showed that the arterial perfusion reserve in patients suffering from PAD is reduced in comparison to that of healthy volunteers (Fig. 8). Further prospective studies are required to evaluate if this technique is sufficient for use in monitoring pro-angiogenic therapy in patients with PAD.

\section{Conclusion}

Muscular diseases and their pathological features are complex. Ultrasound and MRI allow for much more than a simple evaluation of lipomatous, oedematous, hypertrophic or hypotrophic changes within muscular tissue. Pathophysiological surrogate parameters such as muscular microcirculation, sodium homeostasis, energy and lipid metabolism or muscular tissue elasticity can be examined. We expect a much higher level of acceptance of these new techniques in the near future.

\section{References}

1. Zierz S, Jerusalem F (2003) Muskelerkrankungen. Thieme, Stuttgart

2. Reimers CD, Finkenstaedt M (1997) Muscle imaging in inflammatory myopathies. Curr Opin Rheumatol 9:475-485

3. Garcia J (2000) MRI in inflammatory myopathies. Skeletal Radiol 29:425-438
4. Maillard SM, Jones R, Owens C et al (2004) Quantitative assessment of MRI T2 relaxation time of thigh muscles in juvenile dermatomyositis. Rheumatology (Oxford) 43:603-608

5. Mastaglia FL, Garlepp MJ, Phillips BA, Zilko PJ (2003) Inflammatory myopathies: clinical, diagnostic and therapeutic aspects. Muscle Nerve 27:407-425

6. May DA, Disler DG, Jones EA, Balkissoon AA, Manaster BJ (2000) Abnormal signal intensity in skeletal muscle at MR imaging: patterns, pearls, and pitfalls. Radiographics $20 \mathrm{Spec}$ No:S295-S315

7. O'Connell MJ, Powell T, Brennan D, Lynch T, McCarthy CJ, Eustace SJ (2002) Whole-body MR imaging in the diagnosis of polymyositis. AJR Am J Roentgenol 179:967-971

8. Cantwell C, Ryan M, O'Connell M et al (2005) A comparison of inflammatory myopathies at whole-body turbo STIR MRI. Clin Radiol 60:261-267

9. Fleckenstein JL, Reimers CD (1996) Inflammatory myopathies: radiologic evaluation. Radiol Clin North Am 34:427-439, xii

10. Shelly MJ, Bolster F, Foran P, Crosbie I, Kavanagh EC, Eustace SJ (2010) Whole-body magnetic resonance imaging in skeletal muscle disease. Semin Musculoskelet Radiol 14:47-56

11. Hilal SK, Ra JB, Oh CH et al (1988) Sodium imaging. In: Stark DD, Bradley WG (eds) Magnetic resonance imaging. Mosby, St. Louis

12. Nielles-Vallespin S, Weber MA, Bock M et al (2007) 3D radial projection technique with ultrashort echo times for sodium MRI: clinical applications in human brain and skeletal muscle. Magn Reson Med 57:74-81

13. Boada FE, Gillen JS, Shen GX, Chang SY, Thulborn KR (1997) Fast three dimensional sodium imaging. Magn Reson Med 37:706-715

14. Nagel AM, Laun FB, Weber MA, Matthies C, Semmler W, Schad LR (2009) Sodium MRI using a density-adapted 3D radial acquisition technique. Magn Reson Med 62:1565-1573

15. Winter PM, Bansal N (2001) TmDOTP(5-) as a (23)Na shift reagent for the subcutaneously implanted $9 \mathrm{~L}$ gliosarcoma in rats. Magn Reson Med 45:436-442

16. Nagel AM, Weber MA, Matthies C et al (2009) 23Na-MRI contrasts for application in muscular sodium channel diseases. ISMRM 17th Scientific Meeting \& Exhibition, Honolulu, 18-24 April 2009

17. Jurkat-Rott K, Weber MA, Fauler M et al (2009) K+-dependent paradoxical membrane depolarization and $\mathrm{Na}+$ overload, major and reversible contributors to weakness by ion channel leaks. Proc Natl Acad Sci USA 106:4036-4041

18. Weber MA, Nielles-Vallespin S, Huttner HB et al (2006) Evaluation of patients with paramyotonia at $23 \mathrm{Na}$ MR imaging during cold-induced weakness. Radiology 240:489-500

19. Lehmann-Horn F, Jurkat-Rott K (1999) Voltage-gated ion channels and hereditary disease. Physiol Rev 79:1317-1372

20. Weber MA, Nielles-Vallespin S, Essig M, Jurkat-Rott K, Kauczor HU, Lehmann-Horn F (2006) Muscle Na+ channelopathies: MRI detects intracellular $23 \mathrm{Na}$ accumulation during episodic weakness. Neurology 67:1151-1158

21. Chang G, Wang L, Schweitzer ME, Regatte RR (2010) 3D 23Na MRI of human skeletal muscle at 7 Tesla: initial experience. Eur Radiol 20:2039-2046

22. Argov Z, Lofberg M, Arnold DL (2000) Insights into muscle diseases gained by phosphorus magnetic resonance spectroscopy. Muscle Nerve 23:1316-1334

23. McCully KK, Boden BP, Tuchler M, Fountain MR, Chance B (1989) Wrist flexor muscles of elite rowers measured with magnetic resonance spectroscopy. J Appl Physiol 67:926-932

24. Argov Z, Bank WJ, Maris J, Chance B (1987) Muscle energy metabolism in McArdle's syndrome by in vivo phosphorus magnetic resonance spectroscopy. Neurology 37:1720-1724 
25. Gruetter R, Kaelin P, Boesch C, Martin E, Werner B (1990) Noninvasive 31P magnetic resonance spectroscopy revealed McArdle disease in an asymptomatic child. Eur J Pediatr 149:483-486

26. Schroder L, Weber MA, Ulrich M, Regula JU (2006) Metabolic imaging of atrophic muscle tissue using appropriate markers in $1 \mathrm{H}$ and 31P NMR spectroscopy. Neuroradiology 48:809-816

27. Howald H, Boesch C, Kreis R et al (2002) Content of intramyocellular lipids derived by electron microscopy, biochemical assays, and (1)H-MR spectroscopy. J Appl Physiol 92:2264-2272

28. Boesch C, Kreis R (2000) Observation of intramyocellular lipids by $1 \mathrm{H}$-magnetic resonance spectroscopy. Ann N Y Acad Sci 904:25-31

29. Thamer C, Machann J, Bachmann O et al (2003) Intramyocellular lipids: anthropometric determinants and relationships with maximal aerobic capacity and insulin sensitivity. J Clin Endocrinol Metab 88:1785-1791

30. Roden M (2001) Non-invasive studies of glycogen metabolism in human skeletal muscle using nuclear magnetic resonance spectroscopy. Curr Opin Clin Nutr Metab Care 4:261-266

31. Ross B, Lin A, Harris K, Bhattacharya P, Schweinsburg B (2003) Clinical experience with $13 \mathrm{C}$ MRS in vivo. NMR Biomed $16: 358-369$

32. Ledermann HP, Heidecker HG, Schulte AC et al (2006) Calf muscles imaged at BOLD MR: correlation with $\mathrm{TcPO} 2$ and flowmetry measurements during ischemia and reactive hyperemia-initial experience. Radiology 241:477-484

33. Meyer RA, Towse TF, Reid RW, Jayaraman RC, Wiseman RW, McCully KK (2004) BOLD MRI mapping of transient hyperemia in skeletal muscle after single contractions. NMR Biomed 17:392-398

34. Lebon V, Carlier PG, Brillault-Salvat C, Leroy-Willig A (1998) Simultaneous measurement of perfusion and oxygenation changes using a multiple gradient-echo sequence: application to human muscle study. Magn Reson Imaging 16:721-729

35. Donahue KM, Van Kylen J, Guven S et al (1998) Simultaneous gradient-echo/spin-echo EPI of graded ischemia in human skeletal muscle. J Magn Reson Imaging 8:1106-1113

36. Toussaint JF, Kwong KK, Mkparu FO, Weisskoff RM, LaRaia PJ, Kantor HL (1996) Perfusion changes in human skeletal muscle during reactive hyperemia measured by echo-planar imaging. Magn Reson Med 35:62-69

37. Ledermann HP, Schulte AC, Heidecker HG et al (2006) Blood oxygenation level-dependent magnetic resonance imaging of the skeletal muscle in patients with peripheral arterial occlusive disease. Circulation 113:2929-2935

38. Schulte AC, Aschwanden M, Bilecen D (2008) Calf muscles at blood oxygen level-dependent MR imaging: aging effects at postocclusive reactive hyperemia. Radiology 247:482-489

39. Raynaud JS, Duteil S, Vaughan JT et al (2001) Determination of skeletal muscle perfusion using arterial spin labeling NMRI: validation by comparison with venous occlusion plethysmography. Magn Reson Med 46:305-311

40. Wong EC, Buxton RB, Frank LR (1998) A theoretical and experimental comparison of continuous and pulsed arterial spin labeling techniques for quantitative perfusion imaging. Magn Reson Med 40:348-355

41. Frank LR, Wong EC, Haseler LJ, Buxton RB (1999) Dynamic imaging of perfusion in human skeletal muscle during exercise with arterial spin labeling. Magn Reson Med 42:258-267

42. Gardener AG, Gowland PA, Francis ST (2009) Implementation of quantitative perfusion imaging using pulsed arterial spin labeling at ultra-high field. Magn Reson Med 61:874-882

43. Basford JR, Jenkyn TR, An KN, Ehman RL, Heers G, Kaufman KR (2002) Evaluation of healthy and diseased muscle with magnetic resonance elastography. Arch Phys Med Rehabil $83: 1530-1536$

44. Muraki T, Domire ZJ, McCullough MB, Chen Q, An KN (2010) Measurement of stiffness changes in immobilized muscle using magnetic resonance elastography. Clin Biomech (Bristol, Avon) 25:499-503

45. Mlynash M, Olivot JM, Tong DC et al (2009) Yield of combined perfusion and diffusion MR imaging in hemispheric TIA. Neurology 72:1127-1133

46. Hand PJ, Wardlaw JM, Rivers CS et al (2006) MR diffusionweighted imaging and outcome prediction after ischemic stroke. Neurology 66:1159-1163

47. Barrett KM, Ding YH, Wagner DP, Kallmes DF, Johnston KC (2009) Change in diffusion-weighted imaging infarct volume predicts neurologic outcome at 90 days: results of the Acute Stroke Accurate Prediction (ASAP) trial serial imaging substudy. Stroke 40:2422-2427

48. MacKenzie JD, Gonzalez L, Hernandez A, Ruppert K, Jaramillo D (2007) Diffusion-weighted and diffusion tensor imaging for pediatric musculoskeletal disorders. Pediatr Radiol 37:781-788

49. Dietrich O, Raya JG, Sommer J, Deimling M, Reiser MF, BaurMelnyk A (2005) A comparative evaluation of a RARE-based single-shot pulse sequence for diffusion-weighted MRI of musculoskeletal soft-tissue tumors. Eur Radiol 15:772-783

50. Deux JF, Malzy P, Paragios N et al (2008) Assessment of calf muscle contraction by diffusion tensor imaging. Eur Radiol 18:2303-2310

51. Kermarrec E, Budzik JF, Khalil C, Le Thuc V, Hancart-Destee C, Cotten A (2010) In vivo diffusion tensor imaging and tractography of human thigh muscles in healthy subjects. AJR Am J Roentgenol 195:W352-W356

52. Budzik JF, Le Thuc V, Demondion X, Morel M, Chechin D, Cotten A (2007) In vivo MR tractography of thigh muscles using diffusion imaging: initial results. Eur Radiol 17:3079-3085

53. Kan JH, Heemskerk AM, Ding Z et al (2009) DTI-based muscle fiber tracking of the quadriceps mechanism in lateral patellar dislocation. J Magn Reson Imaging 29:663-670

54. Hiltunen J, Suortti T, Arvela S, Seppa M, Joensuu R, Hari R (2005) Diffusion tensor imaging and tractography of distal peripheral nerves at $3 \mathrm{~T}$. Clin Neurophysiol 116:2315-2323

55. Itoh A, Ueno E, Tohno E et al (2006) Breast disease: clinical application of US elastography for diagnosis. Radiology 239:341-350

56. Janssen J, Schlorer E, Greiner L (2007) EUS elastography of the pancreas: feasibility and pattern description of the normal pancreas, chronic pancreatitis, and focal pancreatic lesions. Gastrointest Endosc 65:971-978

57. Janssen J, Dietrich CF, Will U, Greiner L (2007) Endosonographic elastography in the diagnosis of mediastinal lymph nodes. Endoscopy 39:952-957

58. Dock W, Grabenwoger F, Happak W et al (1990) Sonography of the skeletal muscles using high-frequency ultrasound probes. Rofo 152:47-50

59. Krix M, Krakowski-Roosen H, Armarteifio E et al (2011) Comparison of transient arterial occlusion and muscle exercise provocation for assessment of perfusion reserve in skeletal muscle with real-time contrast-enhanced ultrasound. Eur J Radiol 78:419-424

60. Krix M, Weber MA, Kauczor HU, Delorme S, Krakowski-Roosen $\mathrm{H}$ (2009) Changes in the micro-circulation of skeletal muscle due to varied isometric exercise assessed by contrast-enhanced ultrasound. Eur J Radiol 76:110-116

61. Weber MA, Jappe U, Essig M et al (2006) Contrast-enhanced ultrasound in dermatomyositis- and polymyositis. J Neurol 253:1625-1632 